\title{
$\bullet$ \\ Erythroleukoplakia: A High Risk Red-White Premalignant Lesion of the Oral Cavity
}

IJCRR

Section: Healthcare

ISI Impact Factor

(2019-20): 1.628

IC Value (2019): 90.81

$\operatorname{SJIF}(2020)=7.893$

(c) (i) (3)

Copyright@IJCRR

\section{Swain SK ${ }^{1}$, Bhuyan $\mathbf{R}^{2}$, Kar D²}

'Department of Otorhinolaryngology, IMS and SUM Hospital, Siksha “O” Anusandhan University, K8, Kalinganagar, Bhubaneswar-751003, Odisha, India; ${ }^{2}$ Medical Research Laboratory, IMS and SUM Hospital, Siksha “O” Anusandhan University, K8, Kalinganagar, Bhubaneswar-751003, Odisha, India.

\section{ABSTRACT}

Aim: The leukoplakia, erythro-leukoplakia and oral verrucous hyperplasia are premalignant lesions found in the oral cavity. Erythroleukoplakia is an uncommon painless precancerous lesion found in the oral cavity. The erythro-leukoplakia is often seen in the oral mucosa, vermillion and gums. The aetiology of erythro-leukoplakia is often variable and usually associated with cigarette smoking, alcoholism, infections and other agents.

Case Report: A 35-year-old man presented with complaints of burning sensation in the mouth for two months. Oral cavity examinations revealed a reddish-whitish lesion on the right side buccal mucosa. The patient underwent complete surgical excision of the erythro-leukoplakia from the oral cavity.

Results: Biopsy from the lesion is mandatory to rule out malignant transformation and the histopathological examination, in this case, showed dysplastic changes.

Conclusion: The diagnosis of erythro-leukoplakia is based on the clinical presentations and histopathological reports. Clinicians should perform the oral cavity examination properly and alert for the patient's addiction to smoking and alcohol consumption. The treatment of choice is surgery and it should be initiated early for preventing malignant transformation.

Key Words: Erythro-leukoplakia, Leukoplakia, Erythroplakia, Premalignant lesion

\section{INTRODUCTION}

Erythroleukoplakia is a rare premalignant lesion found in the oral mucosa. This lesion shows red and whitish mucosal alterations concomitantly. This premalignant lesion is felt to be at high risk for the development of oral cancer. In erythro-leukoplakia, the red or erythroplakia areas are most commonly demonstrate dysplastic alterations in comparison to the whitish hyperkeratotic areas. ${ }^{1}$ Although leukoplakia or erythroplakia is commonly found premalignant lesion of the oral cavity, but the combined erythro-leukoplakia is an uncommon high-risk precancerous lesion. ${ }^{2}$ The erythro-leukoplakia shows more dysplastic changes in comparison to the whitish leukoplakia area. Although the erythro-leukoplakia is uncommon in clinical practice, have a higher chance of potential towards malignant transformation in the oral cavity, so clinicians should keep this clinical entity in their mind during dealing with such lesions. Here, we present a case of erythro-leukoplakia in the oral cavity of a 35-year-old man.

\section{CASE REPORT}

A 35-year-old male attended the outpatient department of Otorhinolaryngology with complaints of burning sensation in the mouth for two months. He had the habit of betel nut chewing for 10 years. He had also a habit of occasional cigarette smoking and consumption of alcohol. On examination of the oral cavity, it showed a reddish-whitish lesion on the right side buccal mucosa (Fig.1). The clinical diagnosis of erythro-leukoplakia was done. The patient was medicated with an antiseptic gargle, multivitamins and antioxidants for one month. But no change was observed after conservative treatment. The erythro-leukoplakia lesion was completely excised under local anaesthesia and the tissue was sent for histopathological study which revealed dysplastic cells. The patient was advised for cessation of areca nut chewing and also told to completely avoid cigarette smoking and alcohol consumption. The periodic examination of the oral cavity was done which showed no evidence of any recurrence of the lesion.

\section{Corresponding Author:}

Prof. Santosh Kumar Swain, Professor, Department of Otorhinolaryngology, IMS and SUM hospital, Siksha "O" Anusandhan University, K8, Kalinganagar, Bhubaneswar-751003, Odisha, India; Cell: +91-9556524887; Email: santoshswain@soa.ac.in

ISSN: $2231-2196$ (Print)

Received: 14.01 .2021
ISSN: 0975-5241 (Online)

Revised: 15.02 .2021
Accepted: 12.03 .2021
Published: 26.05 .2021 


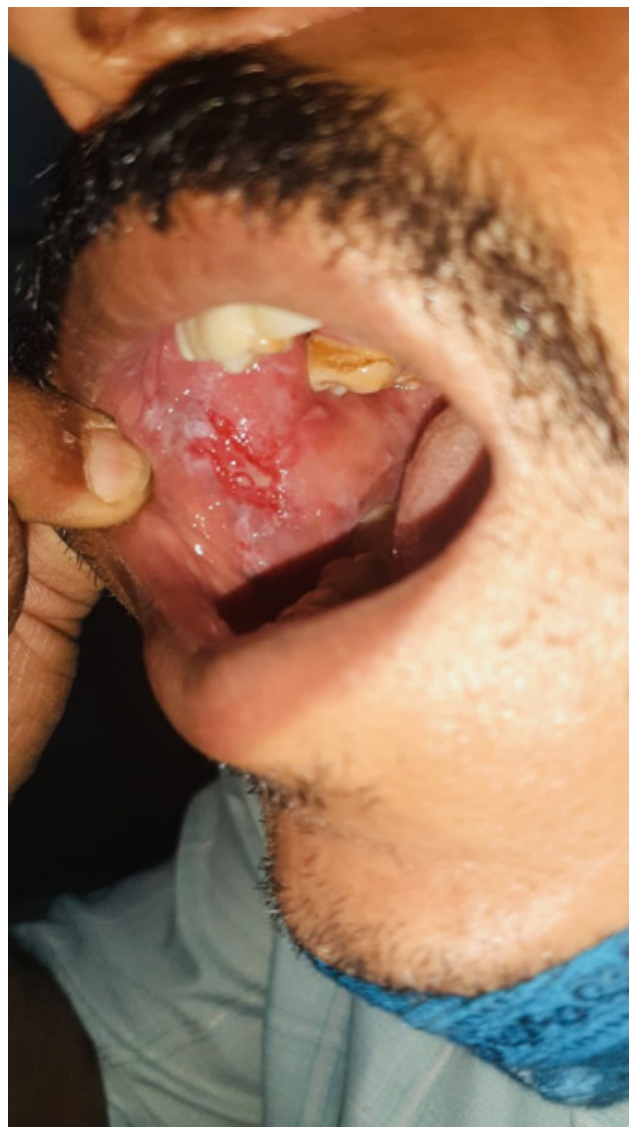

Figure 1: Oral cavity examination showing erythro-leukoplakia in the right side of the oral cavity.

\section{DISCUSSION}

Cancer of the oral cavity is the fifth most common cancer in the world. ${ }^{3}$ The annual incidence of oral cancer is approximately 29,370 cases in the United States. ${ }^{4}$ Oral cancers were the fourth important cause of death among males and sixth among both sexes in all variety of cancers. ${ }^{5}$ Leukoplakia, erythroplakia, erythro-leukoplakia, oral submucosal fibrosis, oral lichen planus and oral candidiasis are common premalignant lesions of the oral cavity. ${ }^{6}$ Erythroleukoplakia is an uncommon premalignant lesion of the oral cavity. One study revealed that the prevalence of erythro-leukoplakia is approximately $11.2 \% .{ }^{7}$ It mainly affects males than females in their seventh decade of life. ${ }^{7}$ In our case report, the patient was an adult male, 35 years of age, addicted to areca nut chewing along with the habit of cigarette smoking and consumption of alcohol.

The common etiologies for causing squamous cell carcinoma are areca quid chewing, cigarette smoking and consumption of alcohol. ${ }^{8}$ Approximately $80 \%$ of oral cancers are associated with these habits. ${ }^{8}$ The oral leukoplakia, erythroplakia and erythro-leukoplakia are important oral precancerous lesions with the potential for malignant transformation. The oral cavity leukoplakia is defined as a whitish patch on the mucosal lining of the oral cavity which cannot be clinically or histopathologically characterized as any other defined disease. ${ }^{9}$ The leukoplakia is an important clinical entity and its diagnosis is based on the exclusion of other lesions as this entity has no such histopathological characteristics. ${ }^{10}$ The buccal mucosa, lower lips and edges of the tongue are commonly affected regions for these premalignant lesions. The erythroplakia has usually a red patch which is often diagnosed clinically and the diagnosis is confirmed by histopathological examination. The lesions of the erythro-leukoplakia are usually different from other pathological lesions and consist of a combination of white and red patches. ${ }^{7}$ The erythro-leukoplakia has a high degree of malignancy in comparison to the oral vanity leukoplakia. ${ }^{7}$

The oral erythro-leukoplakia has a higher chance of malignant transformation than oral leukoplakia. ${ }^{11}$ Histopathologically, around $90 \%$ of the oral leukoplakia shows hyperkeratosis and/or hyperplasia of the epithelial lining whereas nearly all lesions of the oral erythro-leukoplakia show some degree of epithelial dysplasia or carcinoma in situ or superficially invasive carcinoma. ${ }^{11}$ In addition to this, the erythro-leukoplakia of the oral cavity have higher mitotic and apoptotic indices in comparison to the homogenous and nodule oral leukoplakia lesions. ${ }^{12}$ The immunohistochemical study also showed a higher expression of the Ki67 or p53 gene on oral erythro-leukoplakia than leukoplakia of the oral cavity. ${ }^{12}$ One study from Taiwan on areca quid chewing and cigarette smoking leading to progression of premalignant properties of the leukoplakia and erythro-leukoplakia of the oral cavity. ${ }^{13}$ The risks for developing oral cancer with 20 years follow up are $42.2 \%$ for leukoplakia and $95.0 \%$ for erythro-leukoplakia of the oral cavity. ${ }^{14}$ These data suggest that erythroleukoplakia has higher chances of malignant transformation than leukoplakia of the oral cavity.

As per the appearance of the lesion, leukoplakia is divided into 4 types such as early or thin, homogenous or thick, granular or verruciform and spickled or erythro-leukoplakia. Each type of leukoplakia lesion has a different potential for malignant transformation. ${ }^{15}$ The differential diagnosis of the erythro-leukoplakia include chronic hyperplastic candidiasis, lichen planus, leukoderma, reactive hyperkeratosis and spongy white nevus. ${ }^{16}$ The diagnosis is confirmed by the histopathological study where the tissue shows hyperkeratosis, dysplasia, chronic inflammatory infiltrates and acanthosis. Sometimes these lesions show severe dysplasia or carcinoma in situ. ${ }^{17}$ In addition to this, staining by Grocott ruled out the chronic hyperplastic candidiasis. ${ }^{18}$

The traditional treatment of the premalignant lesion of the oral cavity is total surgical excision which may cause a scar formation in case of a large lesion. ${ }^{19}$ Another treatment option is photodynamic therapy which helps to excise the pre- 
malignant lesions of the oral cavity as it can be used repeatedly without cumulative side effects and cause little or no scar formation. ${ }^{20}$ There is the possibility of recurrence of the erythro-leukoplakia after its treatment, so monitoring of the patient should be needed. The important part of the treatment has always advised the patient to stop the habits of betel nut chewing, cigarette smoking and alcohol consumption.

\section{CONCLUSION}

The exact causes of the erythro-leukoplakia are often variable which may be associated with betel nut chewing, cigarette smoking and alcohol consumption. The diagnosis is often done during routine examination of the oral cavity. The diagnosis is based on the clinical presentations and histopathological reports. The specimen is usually collected by the excisional biopsy. The biopsy is usually mandatory to find out the severity of the epithelial dysplasia which act as a decisive factor for further treatment planning. The treatment of the erythro-leukoplakia is complete excision of the lesion. Clinicians should also advise the patients for cessation of areca nut chewing, smoking and alcohol. The treatment should be initiated early for preventing this lesion into a malignant transformation.

\section{ACKNOWLEDGEMENT}

All the authors are thankful to the founder president of the Siksha 'O' Anusandhan University, Prof.Manoj Ranjan Nayak for his constant encouragement.

\section{Conflict of interest: Nil}

Financial support: Nil

Author Contribution: Prof.Santosh Kumar Swain: Concept, data collection and data analysis; Dr.Ruchi Bhuyan: Data collection and drafting; Dr.Dattatreya Kar: Data collection

\section{COMMENTS-}

1. Journal names should be abbreviated in references, references must be in Vancouver style

2. Read all the authors guidelines carefully and revised the manuscript accordingly

\section{REFERENCES}

1. Lockhart PB, Norris CM Jr, Pulliam C. Dental factors in the genesis of squamous cell carcinoma of the oral cavity. Eur J Cancer B Oral Oncol.1998; 34(2):133-139.
2. Swain SK, Debta P.Nonsurgical treatment of oral cavity leukoplakia. Matrix Science Medica 2020;4(4):91-95.

3. Lingen M, Sturgis EM, Kies MS. Squamous cell carcinoma of the head and neck in non-smokers: clinical and biologic characteristics and implications for management. Curr Opin Oncol. 2001; 13(3):176-182.

4. Jemal A, Murray T, Ward E. Cancer statistics. CA Cancer J Clin 2005; 55 (1):10-30.

5. Tsai SP, Wen CP, Hu SC, Cheng TY, Huang SJ. Workplace smoking-related absenteeism and productivity costs in Taiwan. Tobacco control 2005;14(1):33-37.

6. Debta P, Swain SK, Sahu MC, Delta FM, Mohanty JN.A review on oral candida as commensals and opportunistic pathogen. Indian J Med Forensic Med Toxicol2020;14(4):8381-8387.

7. Feller L, Altini M, Slabbert H. Pre-malignant lesions of the oral mucosa in a South African sample - a clinicopathological study. J Dent Assoc S Afr. 1991; 46(5):261-265.

8. Kwan HW. A statistical study on oral carcinomas in Taiwan with emphasis on the relationship with betel nut chewing: a preliminary report. J Formos Med Assoc. 1976; 75(9):497-505.

9. Warnakulasuriya S, Johnson NW, van der Waal I. Nomenclature and classification of potentially malignant disorders of the oral mucosa. J Oral Pathol Med. 2007; 36(10):575-580.

10. Góes C, Weyll B, Sarmento VA, Ramalho LMP. Diagnóstico diferencial e manejo da leucoplasia bucal - Caso clínico: acompanhamento de 4 anos. Res Gyneco Org. 2007; 55(1):95-100.

11. Neville BW, Damm DD, Allen CM, Bouquot JE. Epithelial pathology. In: Neville BW, Damm DD, Allen CM, Bouquot JE, eds. Oral and maxillofacial pathology, 3rd edn, Philadelphia: Sauders Elsevier 2009; 388-398.

12. Kovesi G, Szende B. Changes in apoptosis and mitotic index, p53 and Ki67 expression in various types of oral leukoplakia. Onc. 2003; 65(4): 331-336.

13. Yen AM, Chen SC, Chen TH. Dose-response relationships of oral habits associated with the risk of oral pre-malignant lesions among men who chew betel quid. Oral Oncol. 2007; 43(7): 634638.

14. Yen AM, Chen SC, Chang SH, Chen TH. The effect of betel quid and cigarette on the multistate progression of oral pre-malignancy. J Oral Pathol Med. 2008; 37(7): 417-422.

15. Mortazavi H, Baharvand M, Mehdipour M. Oral potentially malignant disorders: an overview of more than 20 entities. J Dent Res Dent Clin Dent Prosp. 2014; 8(1):6-14.

16. Neville BW, Day TA. Oral cancer and precancerous lesions. CA Cancer J Clin. 2002; 52(4):195-215.

17. Samal S,Delta P,Swain SK.Role of chromium enriched tobacco in the occurrence of oral carcinogenesis.Int J Cur Res Rev. 2020;12(18):20-24.

18. Swain SK,Sahu MC,Debta P,Baisakh MR.Primary fungal laryngitis: An overlooked clinical entity.Apollo Med. 2019;16(1):1115.

19. Vedtofte P, Holmstrup P, Hjorting-Hansen E, Pindborg JJ. Surgical treatment of premalignant lesions of the oral mucosa. Int J Oral Maxillofac Surg. 1987; 16(6): 656-664.

20. Dolmans DE, Fukumura D, Jain RK. Photodynamic therapy for cancer. Nat Rev Cancer. 2003; 3(5): 380-387. 